\title{
Data Logical Structure Design on Teaching Material Management System based on Oracle
}

\author{
Zhe $\mathrm{Li}^{1, \mathrm{a}}$, Hui Ma ${ }^{2, \mathrm{~b}}$ \\ ${ }^{1}$ College of International Exchange, Bohai University, Jinzhou, 121013, China \\ ${ }^{2}$ School of Mathematics and Physics, Bohai University, Jinzhou, 121013, China \\ a503412508@qq.com, bhuima2016@163.com
}

\begin{abstract}
Keywords: Oracle; database; teaching material management system; logical structure design; business processes
\end{abstract}

\begin{abstract}
Teaching material is the knowledge carrier of teaching content and teaching method, and it is the basic tool to carry out teaching work and the important guarantee to improve teaching quality. The construction of scientific and practical teaching materials management system, improve the educational management system in the teaching material management part of the problem, so as to improve work efficiency, improve the level of educational management. This paper provides the basis for the development of the system based on the Oracle database. The research process is based on the business process of teaching material management, the structure of Oracle database, designs data logical structure. The final structure express by logical structure of the 5 tables, which are "teaching plan, teaching material plan, order form, delivery list, and get the teaching materials".
\end{abstract}

\section{Introduction}

Teaching materials, teachers and students are the three basic requirements of classroom teaching activities, but also the three basic elements of teaching quality generation. Teaching materials are the main dependence of teaching and student learning. It is the concrete basis for teachers to teach and do well in teaching and educating people, is an important tool for students to acquire system knowledge, develop intelligence and improve ideological and moral consciousness. According to the characteristics of students' understanding of the general rule and the teaching process in the process of cognition of the students to master the knowledge, usually starting from the teaching materials on perception, perception is more rich, ideas more clearly, the formation of concept and understanding of knowledge are more easily, and teaching materials enable students to obtain knowledge in the learning process more systematic and standardized, have help students to understand and grasp, and is convenient for the students to study, review and homework. From the point of view of the teaching material itself, the teaching material is not only the spokesman of the curriculum standard, but also the professional wisdom and level of many experts and scholars, and it is the essence of the subject knowledge and the crystallization of wisdom. Teaching materials is not a general material and reading materials, is based on the educational purpose and the physical and mental development of students and the characteristics of knowledge, special research and preparation of the text, suitable for the corresponding specific stages of student learning.

Teaching material management is an important part of the educational administration management, doing well the teaching material management is the basic guarantee to carry out the teaching work smoothly, and it is also the inherent requirement of the teaching material. At present, the majority of universities based on the actual situation of the university, have established the material management information system in a different development mode, need of the current teaching management to a certain extent, solve the customer's diversified and personalized services and other aspects of the problem. However, as the leading system and the introduction of the college teaching management departments, lack of professional and technical personnel, the system development and operation management knowledge less, not fully work, cause the system applicability is not strong, resulting in idle resources and even waste, most system did not achieve 
the expected goal. The rapid development of computer technology, storage technology and campus network technology, the traditional information providing and obtaining system has been changed thoroughly, we can make use of the network environment is not affected by time and location information stored, efficient screening and get any information, which makes the solution of the teaching material supply problem has become a possible use of modern information technology.

The construction of scientific and practical teaching materials management system, improve the educational management system in the teaching material management part of the problem, so as to improve work efficiency, improve the level of educational management. Significance in the following three aspects: first, enhance the level of teaching materials management. Using the teaching material management system, the teaching material management work flow, information and automation, not only improve the efficiency of the work. And can be related to the data on the campus network, as long as there is a network where you can handle the relevant work; second is to reduce the burden on the staff. Teaching material management is a complex and hard work, not only need to be patient, but also need to be careful and responsible. If we can use the network system, distribution of the corresponding authority, people in different positions can be in a different place, different time to deal with daily work, it will reduce the pressure on academic staff, avoid struggling to cope with a variety of situations; the third is that the textbook management transparent. All kinds of information on the Internet, management personnel can handle related work by network, students and teachers can view the related material whenever and wherever possible the progress of work, see material cost savings, the use of teaching materials and so on a series of detailed information, transparency contributes to management. In this paper, based on the Oracle database management system, provides basic work for the development of the system.

\section{Business Processes on Teaching Material Management}

System provide services for teachers, students and teaching materials management, manage the whole process of teaching material circulation, is divided into planning management, order management, inventory management and distribution management, etc..

(1) Planning management of teaching materials. Good teaching material planning management, meet the teaching material supply, has an important role for the implementation of teaching plan, stable teaching order, improve the quality of teaching and deepen the teaching reform and so on. In order to make the teaching material plan obey the teaching plan, must study the process of the plan management according to the characteristics of the material supply. Teaching material plan management process as shown in Fig.1.

\begin{tabular}{|c|}
$\begin{array}{c}\text { Teacher personal } \\
\text { plan }\end{array} \rightarrow \begin{array}{c}\text { Plan summary } \\
\text { of teaching and } \\
\text { research section }\end{array}$
\end{tabular}$\rightarrow \begin{gathered}\text { Plan summary } \\
\text { of college }\end{gathered} \rightarrow \begin{gathered}\text { Plan summary } \\
\text { of university }\end{gathered} \rightarrow \begin{gathered}\text { Teaching } \\
\text { material plan } \\
\text { summary table }\end{gathered}$

Fig. 1. Management process on teaching material planning

(2) Order management of teaching materials. According to the summary of teaching materials and teaching center inventory of teaching plans, determine the number of the semester teaching materials used in the deduction of inventory outside need to order, on the teaching of basic information to improve after the treatment, the formation of the order as the original certificate to the supplier ordering materials. Order management process as shown in Fig. 2.

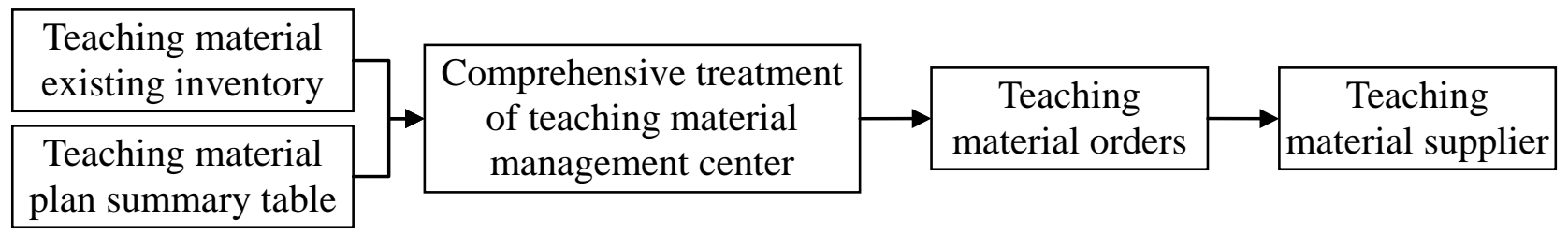

Fig. 2. Management process on teaching material orders

(3) Inventory management of teaching materials. Including warehousing management, inventory 
management and inventory management. After the purchase of materials warehousing, leading to the library before the. Regular inventory of inventory and statistics, on the incomplete, damaged or lost, and so on in a timely manner. Teaching material management center in a timely manner to update the inventory processing and warehousing registration. Inventory management process is shown in Fig. 3.

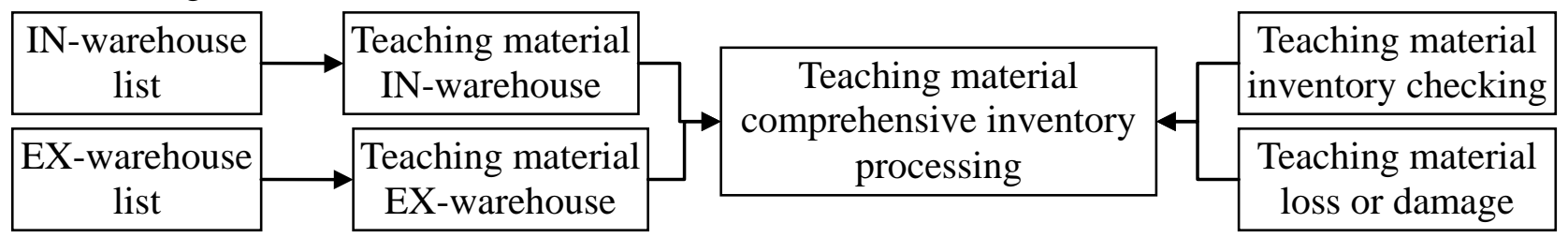

Fig. 3. Management process on teaching material stocks

(4) Distribution of teaching materials. When the ordered materials arrive, the teaching material center according to the teaching plan to inform the college students to receive. According to the class or student individual to provide a list of materials for students to deal with the cost of individual teaching materials, while receiving the materials for inventory processing. Print out a list of details, will be issued to the class or personal materials. Distribution management process as shown in Fig. 4.

\begin{tabular}{|c|c|c|c|}
\hline $\begin{array}{c}\text { Class or personal } \\
\text { teaching materials } \\
\text { issued summary table }\end{array}$ & $\begin{array}{c}\text { Class or personal } \\
\text { receive teaching } \\
\text { material apply }\end{array}$ & $\begin{array}{c}\text { Teaching } \\
\text { material stocks }\end{array}$ & $\begin{array}{c}\text { Class or personal } \\
\text { teaching materials } \\
\text { issuing }\end{array}$ \\
\hline
\end{tabular}

Fig. 4. Management process on teaching material issuing

\section{Oracle Database Architecture}

Oracle database is a relational database management system of Oracle Corp, which provides a set of software products, which is the core of the distributed database. Oracle database is the most popular database system software product. It is a kind of high efficient and reliable database solution. The advantages of [208] embodied in five aspects: advantages, introduces the shared SQL and multi-threaded server system structure, reduce the resource occupation, can support more users on the premise of lower occupied resources; two advantages, provide security management role based on division of labor, has good performance in database management functions integrity check, safety and consistency; three advantages, support a large number of multimedia data, such as binary graphics, sound, animation and other advantages of multidimensional data structure; four, provides a series of interface and software PRO* third generation language, can be in a high level language embedded SQL statements and process (PL/SQL) to the data in the database manipulation; five advantages, provides a new distributed database, can easily read and write remote database data through the network, Symmetric replication technique. The structure of Oracle database is shown in Fig. 5.

The structure of the Oracle database system shown in Figure 5 are described as follows: examples provide a way to access the database, always open a database, and can only open a database; PGA (Program Global Area) is a private memory area of each service process, including memory structure and binding information at run time, is allocated to save session variables in session related information and other areas of memory; SGA (System Global Area) is shared by all services and background processes, the data retrieved from the cache blocks on the disk, the disk cache before the cache redo information, various structures can be shared among users. Parallel query and sharing server mode and some backup operations to support, save the data and Java code specific session Java virtual machine, save buffer CAC The data stored in the he, expired data storage buffer cache, provides cache for database default data block data of different block size; file, including data files, control files and online redo log files and other three categories from the physical point of view including data files, control files redo log files and three files also designed 
the key file to other service for the entire system, such as the parameter file, the password file and archive log files, etc..

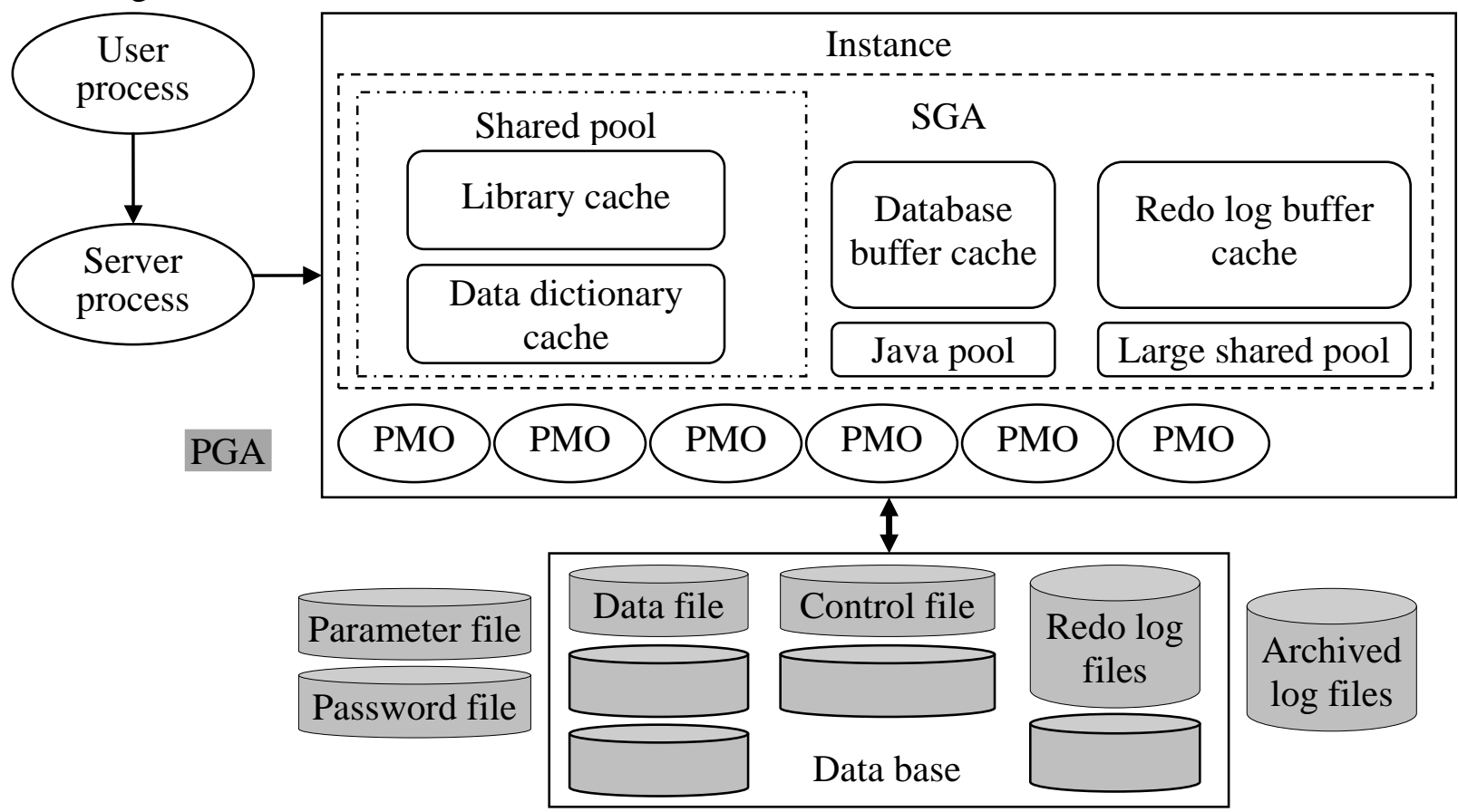

Fig. 5. Oracle database architecture

\section{Data Logical Structure Design}

Database design is the establishment of database and its application system technology, is an important part of software design. Specifically, the database design refers to the application of a given environment, constructing the optimal database model, the establishment of database and its application system, which can effectively store data and meet the application requirements of various users. Database design usually includes three stages: the stage of conceptual design, the database design is the key, through synthesis, induction and abstraction of user requirements, conceptual models to form an independent of a specific database management system; logical design stage, is the concept of data model of structural transfer supported by a database management system, and carries on the optimization; the physical design stage is to select a most suitable application environment of the physical structure of the logic model design is the main storage structure and access methods.

In the Oracle database management system, there are a variety of data types, the design of the logical structure of Table 1 only choose the "CHAR, VARCHAR2, NUMBER, DATE, CLOB, BLOB" and so on six kinds of data types. The CHAR type is used to store fixed length strings; VARCHAR2 type is used to store variable length character strings; NUMBER type is used to store digital data type; DATE type is used to store the date and time data; the CLOB type is used to store a large number of characters; BLOB type is used to store binary files. The logical design results of this paper are as follows:

(1) Teaching plan table: (ID, NUMBER, 10,0), (Course number, CHAR, 10), (Course name, VARCHAR2, 50), (Total hours, NUMBER, 2), (Lecture hours, NUMBER, 2), (Experimental hours, NUMBER, 2), (College name, VARCHAR2, 50), (Professional name, VARCHAR2, 50), (Examination method, VARCHAR2, 20), (Teaching program, BLOB), (Course introduction, CLOB).

(2) Teaching material plan table: (ID, NUMBER, 10,0), (Course number, CHAR, 10), (Use term, CHAR, 11), (Class, CHAR, 2), (Responsible person, VARCHAR2, 20), (Teaching material number, VARCHAR2, 20), (Teaching material name, VARCHAR2, 50), (CIP number, 
VARCHAR2, 20), (Author name, VARCHAR2, 50), (Press, VARCHAR2, 100), (Publication date, DATE, 7), (Unit price, NUMBER, 10,2), (Quantity, NUMBER, 10,0), (Remarks, CLOB).

(3) Purchase order table: (ID, NUMBER, 10,0), (Supplier number, CHAR, 10), (Supplier name, VARCHAR2, 50), (Supplier address, VARCHAR2, 100), (Postcode, CHAR, 10), (Contact, VARCHAR2, 20), (Telephone number, VARCHAR2, 100), (E-mail, VARCHAR2, 100), (QQ, VARCHAR2, 50), (Purchase date, DATE, 7), (Teaching material number, VARCHAR2, 20), (Teaching material name, VARCHAR2, 50), (CIP number, VARCHAR2, 20), (Author name, VARCHAR2, 50), (Press, VARCHAR2, 100), (Publication date, DATE, 7), (Unit price, NUMBER, 10,2), (Quantity, NUMBER, 10,0), (Remarks, CLOB).

(4) Invoice bills table: (ID, NUMBER, 10,0), (Delivery date, DATE, 7), (Delivery quantity, NUMBER, 10,0), (Arrived quantity, NUMBER, 10,0), (non-arrived quantity, NUMBER, 10,0), (Carrier name, VARCHAR2, 100), (Query telephone, VARCHAR2, 30), (Waybill number, VARCHAR2, 30), (Supplier number, CHAR, 10), (Supplier name, VARCHAR2, 50), (Teaching material number, VARCHAR2, 20), (Teaching material name, VARCHAR2, 50), (CIP number, VARCHAR2, 20), (Author name, VARCHAR2, 50), (Press, VARCHAR2, 100), (Publication date, DATE, 7), (Unit price, NUMBER, 10,2), (Quantity, NUMBER, 10,0), (Remarks, CLOB).

(5) Receive teaching material table: (ID, NUMBER, 10,0), (College name, VARCHAR2, 50), (Class, CHAR, 2), (Student number, CHAR, 12), (Student name, VARCHAR2, 50), (Receive date, DATE, 7), (Handlers, VARCHAR2, 50), (Teaching material number, VARCHAR2, 20), (Teaching material name, VARCHAR2, 50), (CIP number, VARCHAR2, 20), (Author name, VARCHAR2, 50), (Press, VARCHAR2, 100), (Publication date, DATE, 7), (Unit price, NUMBER, 10,2), (Quantity, NUMBER, 10,0), (Remarks, CLOB).

\section{References}

[1] J. Qiu, G. Y. Peng, T. W. Pu, et al., "Design and implementation of university teaching material management system based on B/S architecture," Fujian Computer, vol. 31, no. 5, pp. 110-111, 2015.

[2] T. T. Cui, "Research of Web-based Textbook Management System for Colleges and Universities," Computer Knowledge and Technology, vol. 12, no. 6, pp. 56-57, 2016.

[3] Baidu Encyclopedia, "Logical Structure Design on Database," http://baike.baidu.com/link?url=UE8qIaMokFIGIDM-p6YJr-BCm76oXuYPxp5fbzCO6pPG2S BSIYE1u8C9veROPjm7qbLyzppWilC8cyiKuZvc6_, 2015-11-25.

[4] J. Xiong, Y. G. Liu, F. Gao, J. P. Feng, "Research of Oracle Bone Inscriptions Ontology Construction Based on Relational Database," Procedia Environmental Sciences, , vol. 11, no. A, pp. 447-451 2011.

[5] Tom Laszewski, Prakash Nauduri, "Oracle Forms to Oracle Application Development Framework 11g," Elsevier, 2012.

[6] Carrots' blog, "Analysis of the advantages of Oracle Database," http://www.cnblogs.com/muer/archive/2010/04/15/1712719.html, 2016-10-11. 